\title{
Experimental Study of the Strong Interaction at FAIR
}

\section{Diego Bettoni*}

Istituto Nazionale di Fisica Nucleare, Sezione di Ferrara, Ferrara, Italy

E-mail: bettoni@fe.infn.it

The physics program of the future FAIR facility covers a wide range of topics that address central issues of strong interactions and QCD. The antiproton beam of unprecedented quality in the momentum range from $1 \mathrm{GeV} / \mathrm{c}$ to $15 \mathrm{GeV} / \mathrm{c}$ will allow to make high precision, high statistics measurements, from charmonium spectroscopy to the search for exotic hadrons and the study of nucleon structure, from the study of in-medium modifications of hadron masses to the physics of hypernuclei.

In addition to that the possibility to polarize antiprotons will provide access to a wealth of singleand double-spin observables, opening up the possibility to perform new, unique measurements, like the transversity distribution of the valence quarks in the proton, new measurements of singlespin asymmetries and the first determination of the relative phase of the electric and magnetic form factors of the proton in the time-like region.

In this paper we will discuss the experimental program of the PANDA and PAX experiments, which will address these fundamental issues at FAIR.

The XXV International Symposium on Lattice Field Theory

July 30-4 August 2007

Regensburg, Germany

\footnotetext{
* Speaker.
} 


\section{Introduction}

One of the most challenging and fascinating goals of modern physics is the achievement of a fully quantitative understanding of the strong interaction, which is the subject of hadron physics. Significant progress has been achieved over the past few years thanks to considerable advances in experiment and theory. New experimental results have stimulated a very intense theoretical activity and a refinement of the theoretical tools.

Still there are many fundamental questions which remain basically unanswered. Phenomena such as the confinement of quarks, the existence of glueballs and hybrids, the origin of the masses of hadrons in the context of the breaking of chiral symmetry are long-standing puzzles and represent the intellectual challenge in our attempt to understand the nature of the strong interaction and of hadronic matter.

Experimentally, studies of hadron structure can be performed with different probes such electrons, pions, kaons, protons or antiprotons. In antiproton-proton annihilation particles with gluonic degrees of freedom as well as particle-antiparticle pairs are copiously produced, allowing spectroscopic studies with very high statistics and precision. Therefore, antiprotons are an excellent tool to address the open problems.

In addition, the availability of polarized antiprotons would provide access to numerous spin observables, offering further opportunities to perform new, unique measurements which would significantly improve our knowledge of the spin structure of the nucleon.

The recently approved FAIR facility (Facility for Antiproton and Ion Research), which will be built as a major upgrade of the existing GSI laboratory in Germany, will provide antiproton beams of the highest quality in terms of intensity and resolution, which will provide an excellent tool to answer these fundamental questions.

The PANDA experiment (Pbar ANnihilations at DArmstadt) will use the antiproton beam from the High-Energy Storage Ring (HESR) colliding with an internal proton target and a general purpose spectrometer to carry out a rich and diversified hadron physics program, from charmonium spectroscopy to the search for exotic hadrons and the study of nucleon structure, from the study of in-medium modifications of hadron masses to the physics of hypernuclei.

Once a polarized antiproton beam becomes available the PAX experiment (Polarized Antiproton eXperiments), which will be installed also at the HESR, will be able to carry out measurements such as the transversity distribution of the valence quarks in the proton, new measurements of single-spin asymmetries and the first determination of the relative phase of the electric and magnetic form factors of the proton in the time-like region.

This paper is organized as follows: in section 2 we will give an overview of the FAIR facility and the HESR; in section 3 we will discuss some of the most significant items of the PANDA experimental program; in section 4 we will give a brief description of the PANDA detector. Section 5 will be devoted to the PAX scientific program; section 6 will give an overview of the PAX experimental setup, whereas section 7 will briefly deal with the question of antiproton polarization. Finally in section 8 we will present our conclusions. 


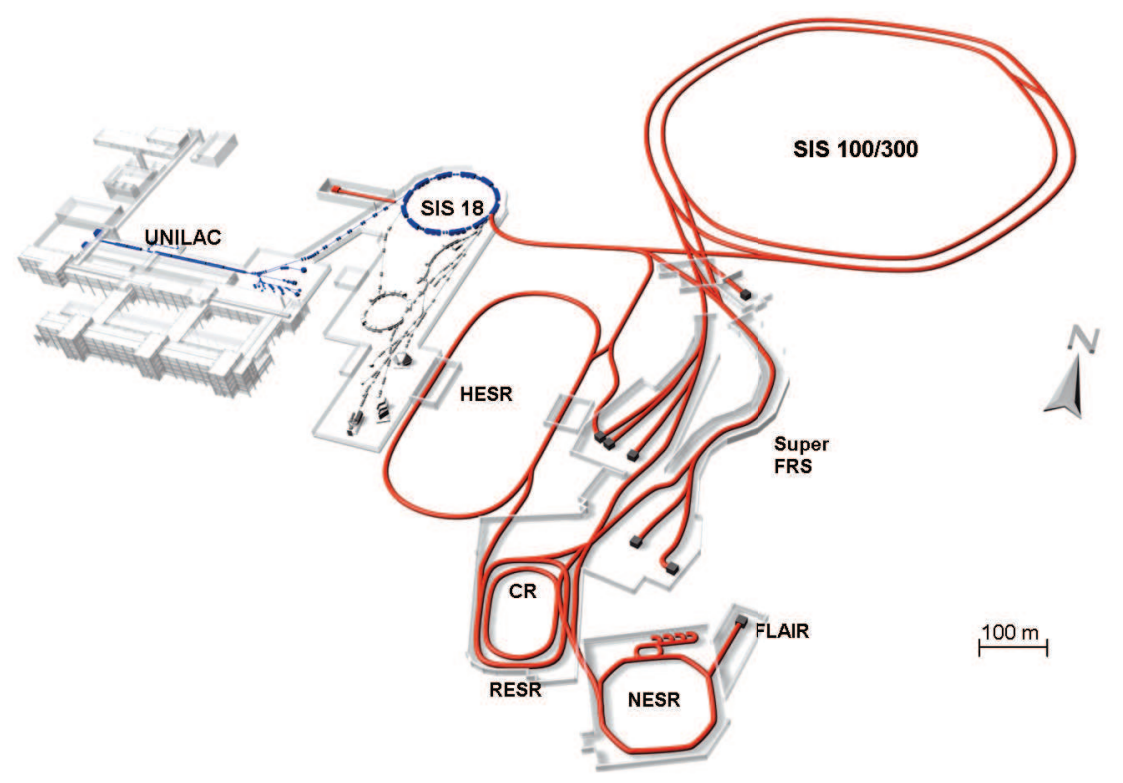

Figure 1: The FAIR complex.

\section{The FAIR facility}

The planned FAIR complex is shown in Fig. 1. The heart of the system consists of two synchrotron rings, called SIS100 and SIS300, housed in the same tunnel, which will provide proton and ion beams of unprecedented quality. The SIS100, a $100 \mathrm{t} \cdot \mathrm{m}$ proton ring, will feed the radioactive ion and antiproton beam lines for experiments to be carried out in the High-Energy Storage Ring (HESR), the Collector and Cooler rings (CR) and the New Experimental Storage Ring (NESR). The SIS300 will deliver high energy ion beams for the study of ultra relativistic heavy ion collisions.

The accelerators of FAIR will feature significant improvements in system parameters over existing facilities:

- beam intensity: increased by a factor of 100 to 1000 for primary and 10000 for secondary beams;

- beam energy will increase by a factor 30 for heavy ions;

- beam variety: FAIR will offer a variety of beam lines, from antiprotons to protons, to uranium and radioactive ions;

- beam precision: availability of cooled antiproton and ion beams (stochastic and electron cooling);

- parallel operation: full accelerator performance for up to four different, independent experiments and experimental programs.

These features will make FAIR a first rate facility for experiments in particle, nuclear, atomic, plasma and applied physics. 


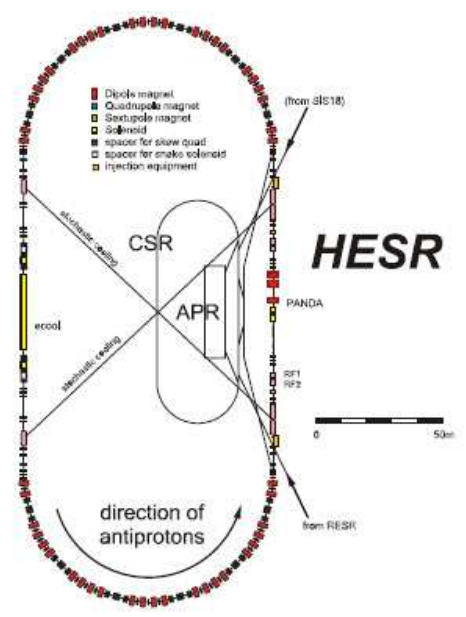

Figure 2: Layout of the HESR.

\subsection{The High-Energy Storage Ring}

The antiproton beam will be produced by a primary proton beam from the SIS100. The $\bar{p}$ production rate will be of approximately $2 \times 10^{7} / \mathrm{s}$. After $5 \times 10^{5} \bar{p}$ have been produced they will be transferred to the HESR, where internal experiments in the $\bar{p}$ momentum range from $1 \mathrm{GeV} / \mathrm{c}$ to $15 \mathrm{GeV} / \mathrm{c}$ can be performed.

The layout of the HESR is shown in Fig. 2. It is a racetrack ring, 574 meters in length, with two straight sections which will host the electron cooling and, respectively, the PANDA (and PAX) experiments. Two modes of operation are foreseen: in the high-luminosity mode peak luminosities of $2 \times 10^{32} \mathrm{~cm}^{-2} \mathrm{~s}^{-1}$ will be reached with a beam momentum spread $\delta p / p=10^{-4}$, achieved by means of stochastic cooling; in the high-resolution mode for beam momenta below $8 \mathrm{GeV} / \mathrm{c}$ electron cooling will yield a smaller beam momentum spread $\delta p / p=10^{-5}$ at a reduced luminosity of $10^{31} \mathrm{~cm}^{-2} \mathrm{~s}^{-1}$. The high-resolution mode will allow to measure directly the total width of very narrow (below $1 \mathrm{MeV}$ ) resonances.

\section{The PANDA Physics Program}

The PANDA experiment has a rich experimental program whose ultimate aim is to improve our knowledge of the strong interaction and of hadron structure. The experiment is being designed to fully exploit the extraordinary physics potential arising from the availability of high-intensity, cooled antiproton beams. Significant progress beyond the present understanding of the field is expected thanks to improvements in statistics and precision of the data.

Many experiments are foreseen in PANDA. In this paper we will discuss the following:

- charmonium spectroscopy;

- search for gluonic excitations (hybrids and glueballs);

- study of hadrons in nuclear matter; 
- spectroscopy of single and double $\Lambda$-hypernuclei;

- measurement of the electromagnetic form factors of the proton in the time-like region.

\subsection{Charmonium Spectroscopy}

Ever since its discovery in 1974 [1] charmonium has been a powerful tool for the understanding of the strong interaction. The high mass of the $c$ quark $\left(\mathrm{m}_{c} \approx 1.5 \mathrm{GeV} / \mathrm{c}^{2}\right)$ makes it plausible to attempt a description of the dynamical properties of the $(c \bar{c})$ system in terms of non-relativistic potential models, in which the functional form of the potential is chosen to reproduce the asymptotic properties of the strong interaction. The free parameters in these models are to be determined from a comparison with the experimental data.

Now, more than thirty years after the $J / \psi$ discovery, charmonium physics continues to be an exciting and interesting field of research. The recent discoveries of new states $\left(\eta_{c}^{\prime}, \mathrm{X}(3872)\right)$, and the exploitation of the B factories as rich sources of charmonium states have given rise to renewed interest in heavy quarkonia, and stimulated a lot of experimental and theoretical activities. Over the past few years a significant progress has been achieved by Lattice Gauge Theory calculations, which have become increasingly more capable of dealing quantitatively with non perturbative dynamics in all its aspects, starting from the first principles of QCD.

\subsubsection{Experimental Study of Charmonium}

Experimentally charmonium has been studied mainly in $e^{+} e^{-}$and $\bar{p} p$ experiments.

In $e^{+} e^{-}$annihilations direct charmonium formation is possible only for states with the quantum numbers of the photon $J^{P C}=1^{--}$, namely the $J / \psi, \psi^{\prime}$ and $\psi(3770)$ resonances. Precise measurements of the masses and widths of these states can be obtained from the energy of the electron and positron beams, which are known with good accuracy. All other states can be reached by means of other production mechanisms, such as photon-photon fusion, initial state radiation, B-meson decay and double charmonium.

On the other hand all $c \bar{c}$ states can be directly formed in $\bar{p} p$ annihilations, through the coherent annihilation of the three quarks in the proton with the three antiquarks in the antiproton. This technique, originally proposed by P. Dalpiaz in 1979 [2], could be successfully employed a few years later at CERN and Fermilab thanks to the development of stochastic cooling. With this method the masses and widths of all charmonium states can be measured with excellent accuracy, determined by the very precise knowledge of the initial $\bar{p} p$ state and not limited by the resolution of the detector.

The cross section for the process:

$$
\bar{p} p \rightarrow(\bar{c} c) \rightarrow \text { final state }
$$

is given (in units $\hbar=c=1$ ) by the well known Breit-Wigner formula:

$$
\sigma_{B W}(E)=\frac{2 J+1}{4} \frac{\pi}{k^{2}} \frac{B_{\text {in }} B_{\text {out }} \Gamma_{R}^{2}}{\left(E-M_{R}\right)^{2}+\Gamma_{R}^{2} / 4}
$$

where $E$ and $k$ are the center-of-mass (c.m.) energy and momentum; $J, M_{R}$ and $\Gamma_{R}$ are the resonance spin, mass and total width and $B_{\text {in }}$ and $B_{\text {out }}$ are the branching ratios into the initial $(\bar{p} p)$ and final 
states. Due to the finite energy spread of the beam, the measured cross section is a convolution of the Breit-Wigner cross section, eq. (3.2), and the beam energy distribution function $f\left(E, \Delta E_{B}\right)$; the effective production rate $v$ is given by:

$$
v=L_{0}\left\{\varepsilon \int d E f\left(E, \Delta E_{B}\right) \sigma_{B W}(E)+\sigma_{b}\right\}
$$

where $L_{0}$ is the instanteneous luminosity, $\varepsilon$ an overall efficiency $\times$ acceptance factor and $\sigma_{b}$ a background term.

The parameters of a given resonance can be extracted by measuring the formation rate for that resonance as a function of the c.m. energy $E_{c m}$. The accurate determination of masses and widths depends crucially on the precise knowledge of the absolute energy scale and on the beam energy spectrum.
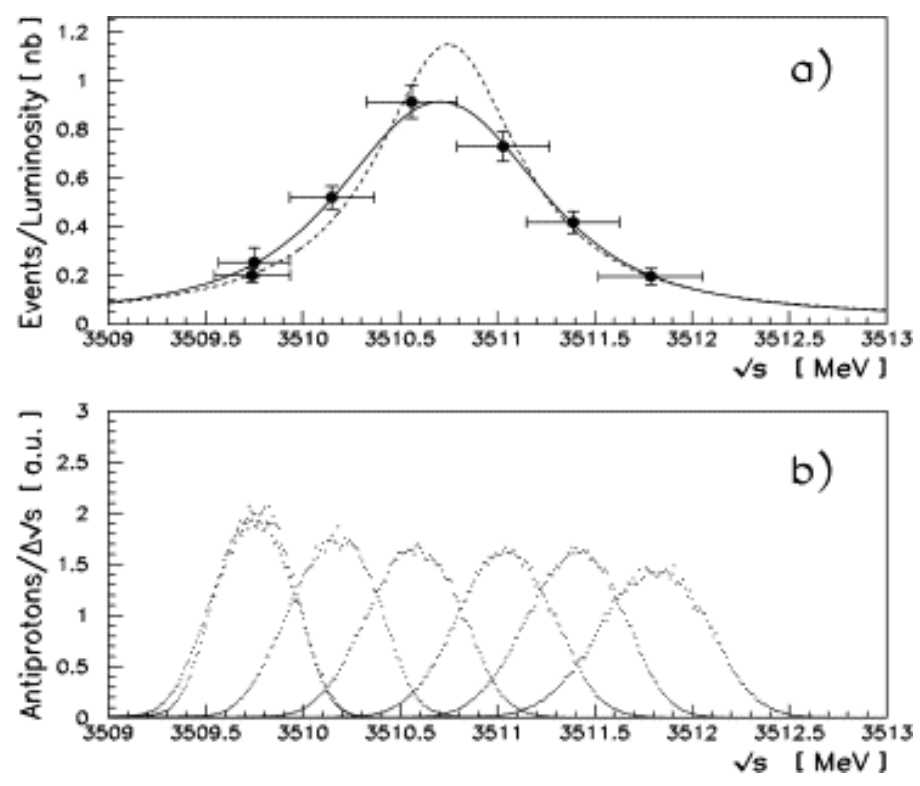

Figure 3: Resonance scan at the $\chi_{c 1}$ carried out at Fermilab (a) and beam energy distribution in each data point (b).

The technique is illustrated in Fig. 3 which shows a scan of the $\chi_{c 1}$ resonance carried out at the Fermilab antiproton accumulator by the E835 experiment [3] using the process $\bar{p} p \rightarrow \chi_{c 1} \rightarrow J / \psi \gamma$. For each point of the scan the horizontal error bar in (a) corresponds to the width of the beam energy distribution. The actual beam energy distribution is shown in (b). This scan allowed the E835 experiment to carry out the most precise measurement of the mass $(3510.719 \pm 0.051 \pm 0.019$ $\left.\mathrm{Mev} / \mathrm{c}^{2}\right)$ and total width $(0.876 \pm 0.045 \pm 0.026 \mathrm{MeV})$ of this resonance.

\subsubsection{The charmonium spectrum}

The spectrum of charmonium states is shown in Fig. 4. It consists of eight narrow states below the open charm threshold $(3.73 \mathrm{GeV})$ and several tens of states above the threshold.

All eight states below $D \bar{D}$ threshold are well established, but whereas the triplet states are measured with very good accuracy, the same cannot be said for the singlet states. 


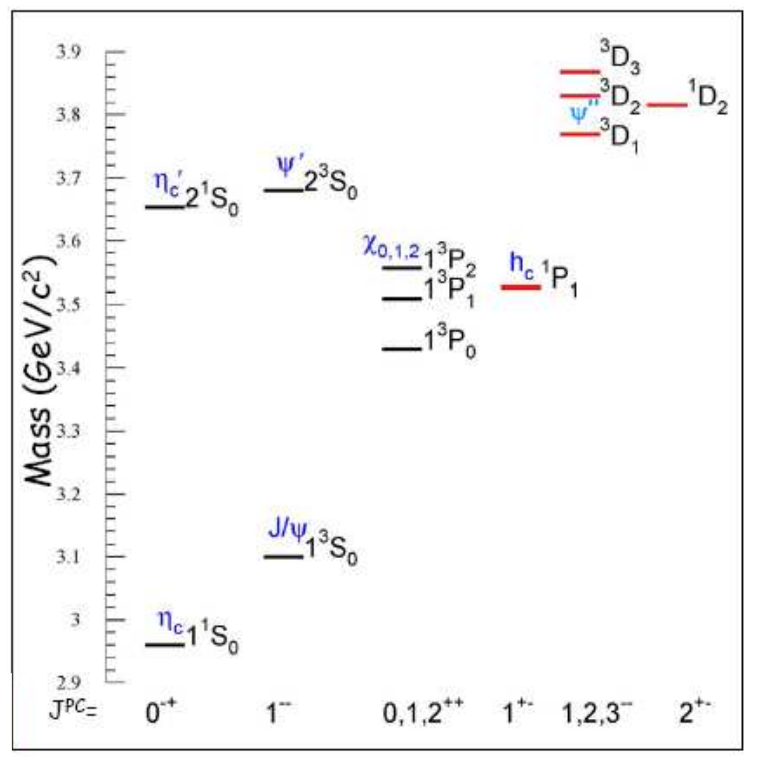

Figure 4: The charmonium spectrum.

The $\eta_{c}$ was discovered almost thirty years ago and many measurements of its mass and total width exist, with six new measurements in the last four years. Still the situation is far from satisfactory. The Particle Data Group (PDG) [4] value of the mass is $2980.4 \pm 1.2 \mathrm{MeV} / \mathrm{c}^{2}$, an average of eight measurements with an internal confidence level of 0.026: the error on the $\eta_{c}$ mass is still as large as $1.2 \mathrm{MeV} / \mathrm{c}^{2}$, to be compared with few tens of $\mathrm{KeV} / \mathrm{c}^{2}$ for the $J / \psi$ and $\psi^{\prime}$ and few hundreds of $\mathrm{KeV} / \mathrm{c}^{2}$ for the $\chi_{c J}$ states. The situation is even worse for the total width: the PDG average is $25.5 \pm 3.4 \mathrm{MeV}$, with an overall confidence level of only 0.001 and individual measurements ranging from $7 \mathrm{MeV}$ to $34.3 \mathrm{MeV}$. The most recent measurements have shown that the $\eta_{c}$ width is larger than was previously believed, with values which are difficult to accomodate in quark models. This situation points to the need for new high-precision measurements of the $\eta_{c}$ parameters.

The first experimental evidence of the $\eta_{c}(2 S)$ was reported by the Crystal Ball collaboration [5], but this finding was not confirmed in subsequent searches in $\bar{p} p$ or $e^{+} e^{-}$experiments. The $\eta_{c}(2 S)$ was finally discovered by the Belle collaboration [6] in the hadronic decay of the B meson $B \rightarrow K+\eta_{c}(2 S) \rightarrow K+\left(K_{s} K^{-} \pi^{+}\right)$with a mass which was incompatible with the Crystal Ball candidate. The Belle finding was then confirmed by CLEO [7] and BaBar [8] which observed this state in two-photon fusion. The PDG value of the mass is $3638 \pm 4 \mathrm{MeV} / \mathrm{c}^{2}$, corresponding to a surprisingly small hyperfine splitting of $48 \pm 4 \mathrm{MeV} / \mathrm{c}^{2}$, whereas the total width is only measured with an accuracy of 50\%. The study of this state has just started and all its properties need to be measured with good accuracy.

The ${ }^{1} P_{1}$ state of charmonium $\left(h_{c}\right)$ is of particular importance in the determination of the spindependent component of the $q \bar{q}$ confinement potential. The Fermilab experiment E760 reported an $h_{c}$ candidate in the decay channel $J / \psi \pi^{0}$ [9], with a mass of $3526.2 \pm 0.15 \pm 0.2 \mathrm{MeV} / \mathrm{c}^{2}$. This finding was not confirmed by the successor experiment E835, which however observed an enhancement in the $\eta_{c} \gamma$ [10] final state at a mass of $3525.8 \pm 0.2 \pm 0.2 \mathrm{MeV} / \mathrm{c}^{2}$. The $h_{c}$ was 
finally observed by the CLEO collaboration [11] in the process $e^{+} e^{-} \rightarrow \psi^{\prime} \rightarrow h_{c}+\pi^{0}$ with $h_{c} \rightarrow$ $\eta_{c}+\gamma$, in which the $\eta_{c}$ was identified via its hadronic decays. They found a value for the mass of $3524.4 \pm 0.6 \pm 0.4 \mathrm{MeV} / \mathrm{c}^{2}$. It is clear that the study of this state has just started and that many more measurements will be needed to determine its properties, in particular the width.

The region above $D \bar{D}$ threshold is rich in interesting new physics. In this region, close to the $D \bar{D}$ threshold, one expects to find the four 1D states. Of these only the $1^{3} D_{1}$, identified with the $\psi(3770)$ resonance, has been found. The $J=2$ states $\left(1^{1} D_{2}\right.$ and $\left.1^{3} D_{2}\right)$ are predicted to be narrow, because parity conservation forbids their decay to $D \bar{D}$. In addition to the $\mathrm{D}$ states, the radial excitations of the $\mathrm{S}$ and $\mathrm{P}$ states are predicted to occur above the open charm threshold. None of these states have been positively identified.

The experimental knowledge of this energy region comes from data taken at the early $e^{+} e^{-}$ experiments at SLAC and DESY and, more recently, at the B-factories, CLEO-c and BES. The structures and the higher vector states observed by the early $e^{+} e^{-}$experiments have not all been confirmed by the latest much more accurate measurements by BES [12]. A lot of new states have recently been discovered at the $\mathrm{B}$-factories, mainly in the hadronic decays of the meson: these new states $(\mathrm{X}, \mathrm{Y}, \mathrm{Z}$...) are associated with charmonium because they decay predominantly into charmonium states such as the $J / \psi$ or the $\psi^{\prime}$, but their interpretation is far from obvious. The situation can be roughly summarized as follows:

- the $\mathrm{Z}(3931)$ [13], observed in two-photon fusion and decaying predominantly into $D \bar{D}$, is tentatively identified with the $\chi_{c 2}(2 S)$;

- the $X(3940)$ [14], observed in double charmonium events, is tentatively identified with the $\eta_{c}(3 \mathrm{~S})$

- for all other new states (X(3872), Y(3940), Y(4260), Y(4320) and so on) the interpretation is not at all clear, with speculations ranging from the missing $c \bar{c}$ states, to molecules, tetraquark states, and hybrids. It is obvious that further measurements are needed to determine the nature of these new resonances.

The main challenge of the next years will be thus to understand what these new states are and to match these experimental findings to the theoretical expectations for charmonium above threshold.

\subsubsection{Charmonium in PANDA}

Charmonium spectroscopy is one of the main items in the experimental program of PANDA, and the design of the detector and of the accelerator are optimized to be well suited for this kind of physics. PANDA will represent a substantial improvement over the Fermilab experiments E760 and E835:

- up to ten times higher instantaneous luminosity $\left(\mathscr{L}=2 \times 10^{32} \mathrm{~cm}^{-2} \mathrm{~s}^{-1}\right.$ in high-luminosity mode, compared to $2 \times 10^{31} \mathrm{~cm}^{-2} \mathrm{~s}^{-1}$ at Fermilab);

- better beam momentum resolution $\left(\Delta p / p=10^{-5}\right.$ in high-resolution mode, compared with $10^{-4}$ at Fermilab); 
- a better detector (higher angular coverage, magnetic field, ability to detect the hadronic decay modes).

At full luminosity PANDA will be able to collect several thousand $c \bar{c}$ states per day. By means of fine scans it will be possible to measure masses with accuracies of the order of $100 \mathrm{KeV}$ and widths to $10 \%$ or better. The entire energy region below and above open charm threshold will be explored.

\subsection{Gluonic Excitations}

One of the main challenges of hadron physics, and an important item in the PANDA physics program, is the search for gluonic excitations, i.e. hadrons in which the gluons can act as principal components. These gluonic hadrons fall into two main categories: glueballs, i.e. states of pure glue, and hybrids, which consist of a $q \bar{q}$ pair and excited glue. The additional degrees of freedom carried by gluons allow these hybrids and glueballs to have $J^{P C}$ exotic quantum numbers: in this case mixing effects with nearby $q \bar{q}$ states are excluded and this makes their experimental identification easier. The properties of glueballs and hybrids are determined by the long-distance features of QCD and their study will yield fundamental insight into the structure of the QCD vacuum.

Antiproton-proton annihilations provide a very favourable environment in which to look for gluonic hadrons. Two particles, first seen in $\pi N$ scattering [15] with exotic quantum numbers $J^{P C}=1^{-+}, \pi_{1}(1400)$ [16] and $\pi_{1}(1600)$ [17], are clearly seen in $\bar{p} p$ annihilation at rest. On the other hand a narrow state at $1500 \mathrm{MeV} / \mathrm{c}^{2}$ discovered in $\bar{p} p$ annihilations by the Crystal Barrel experiment [18], is considered the best candidate for the glueball ground state $\left(J^{P C}=0^{++}\right)$, even though the mixing with nearby $q \bar{q}$ states makes this interpretation difficult.

So far the experimental search for glueballs and hybrids has been mainly carried out in the mass region below $2.2 \mathrm{MeV} / \mathrm{c}^{2}$. PANDA will extend the search to higher masses and in particular to the charmonium mass region, were light quark states form a structure-less continuum and heavy quark states are far fewer in number. Therefore exotic hadrons in this mass region could be resolved and identified unambiguously.

\subsubsection{Charmonium Hybrids}

The spectrum of charmonium hybrid mesons can be calculated within the framework of various theoretical models, such as the bag model, the flux tube model, the constituent quark model and recently, with increasing precision, from Lattice QCD (LQCD). For these calculations the parameters are fixed according to the properties of the known $q \bar{q}$ states. All model predictions and LQCD calculations agree that the masses of the lowest lying charmonium hybrids are between $4.2 \mathrm{GeV} / \mathrm{c}^{2}$ and $4.5 \mathrm{GeV} / \mathrm{c}^{2}$. Three of these states are expected to have $J^{P C}$ exotic quantum numbers $\left(0^{+-}\right.$, $\left.1^{-+}, 2^{+-}\right)$, making their experimental identification easier since they will not mix with nearby $c \bar{c}$ states. These states are expected to be narrower than conventional charmonium, because their decay to open charm will be suppressed or forbidden below the $D \overline{D_{J}^{*}}$ threshold. The cross sections for the formation and production of charmonium hybrids are estimated to be similar to those of normal charmonium states, which are within experimental reach. Formation experiments will generate only non-exotic charmonium hybrids, whereas production experiments will yield both exotic 
and non-exotic states. This feature can be exploited experimentally: the observation of a state in production but not in formation will be, in itself, a strong hint of exotic behavior.

\subsubsection{Glueballs}

The glueball spectrum can be calculated within the framework of LQCD in the quenched approximation [19]. In the mass range accessible to PANDA as many as 15 glueball states are predicted, some with exotic quantum numbers (oddballs). As with hybrids, exotic glueballs are easier to identify experimentally since they do not mix with conventional mesons. The complications arising from mixing with normal $q \bar{q}$ states is well illustrated by the case of the $\mathrm{f}_{0}(1500)$. As mentioned above, this narrow state, observed at LEAR by the Crystal Barrel [18] and Obelix [20] experiments, is considered the best candidate for the ground state glueball. However this interpretation is not unique, and relies on the combined analysis of the complete set of two-body decays of the $\mathrm{f}_{0}(1500)$ and two other scalar states, the $\mathrm{f}_{0}(1370)$ and the $\mathrm{f}_{0}(1710)$. This analysis yields the following mixing picture [21]:

$$
\begin{array}{r}
\left|f_{0}(1710)>=0.39\right| g g>+0.91|s \bar{s}>+0.14| N \bar{N}> \\
\left|f_{0}(1500)>=-0.69\right| g g>+0.37|s \bar{s}>-0.62| N \bar{N}> \\
\left|f_{0}(1370)>=0.60\right| g g>-0.13|s \bar{s}>-0.79| N \bar{N}>
\end{array}
$$

where $\mid N \bar{N}>=(|u \bar{u}>+| d \bar{d}>) / \sqrt{2}$. Other scenarios for the scalar meson nonet not involving a glueball have been proposed and this makes the interpretation of the $\mathrm{f}_{0}(1500)$ as the ground state glueball ambiguous. This example highlights the need to extend the glueball search to higher mass regions, which are free of the problem of mixing with conventional $q \bar{q}$ states.

\subsection{Hadrons in Nuclear Matter}

The study of medium modifications of hadrons embedded in hadronic matter is aimed at understanding the origin of hadron masses in the context of spontaneous chiral symmetry breaking in QCD and its partial restoration in a hadronic environment. So far experiments have been focussed on the light quark sector: evidence of mass changes for pions and kaons have been deduced by the study of deeply bound pionic atoms [22] and of $\mathrm{K}$ meson production in proton-nucleus and heavy-ion collisions [23].

The high-intensity $\bar{p}$ beam of up to $15 \mathrm{GeV} / \mathrm{c}$ will allow an extension of this program to the charm sector both for hadrons with hidden and open charm. The in-medium masses of these states are expected to be affected primarily by the gluon condensate. Recent theoretical calculations predict small mass shifts (5-10 MeV/c ${ }^{2}$ ) for the low-lying charmonium states [24] and more consistent effects for the $\chi_{c J}\left(40 \mathrm{MeV} / \mathrm{c}^{2}\right), \psi^{\prime}\left(100 \mathrm{MeV} / \mathrm{c}^{2}\right)$ and $\psi(3770)\left(140 \mathrm{MeV} / \mathrm{c}^{2}\right)$ [25].

$\mathrm{D}$ mesons, on the other hand, offer the unique opportunity to study the in-medium dynamics of a system with a single light quark. Recent theoretical calculations agree in the prediction of a mass splitting for D mesons in nuclear matter but, unfortunately, they disagree in sign and size of the effect.

Experimentally the in-medium masses of charmonium states can be reconstructed from their decay into di-leptons and photons, which are not affected by final state interaction. D meson masses, on the other hand, need to be reconstructed by their weak decays into pions and kaons 
which makes the direct measurement of mass modifications difficult. Therefore other signals have been proposed for the detection of in-medium mass shifts of $\mathrm{D}$ mesons: in particular it has been speculated that a lowering of the $D \bar{D}$ threshold would result in an increased $D$ and $\bar{D}$ production in $\bar{p}$-nucleus annihilations [26] or in an increase in width of the charmonium states lying close to the threshold [27].

Another study which can be carried out in PANDA is the measurement of $J / \psi$ and D meson production cross sections in $\bar{p}$ annihilation on a series of nuclear targets. The comparison of the resonant $J / \psi$ yield obtained from $\bar{p}$ annihilation on protons and different nuclear targets allows to deduce the $J / \psi$-nucleus dissociation cross section, a fundamental parameter to understand $J / \psi$ suppression in relativistic heavy ion collisions interpreted as a signal for quark-gluon plasma formation.

\subsection{Hypernuclear Physics}

Hypernuclei are systems in which up or down quarks are replaced by strange quarks. In this way a new quantum number, strangeness, is introduced into the nucleus. Although single [28] and double [29] $\Lambda$-hypernuclei were discovered many decades ago, only 6 double $\Lambda$-hypernuclei are presently known. The availability of $\bar{p}$ beams at FAIR will allow efficient production of hypernuclei with more than one strange hadron, making PANDA competitive with planned dedicated facilities. This will open new perspectives for nuclear structure spectroscopy and for studying the forces between hyperons and nucleons.

The production of double $\Lambda$-hypernuclei in PANDA will be achieved by means of a multistep process:

- using an energetic $\bar{p}$ beam a $\Xi^{-}$(together with its associated strange particles) is produced in a primary nuclear target;

- the produced $\Xi^{-}$is slowed down in a dense, solid material (e.g. a nuclear emulsion) and forms a $\Xi^{-}$atom;

- after an atomic cascade the hyperon is finally captured by a secondary target nucleus, leading to the formation of a double hypernucleus via the process $\Xi^{-} p \rightarrow \Lambda \Lambda$.

The spectroscopic information on double hypernuclei will be obtained by detecting the $\gamma$-rays emitted in the decay of the excited hypernucleus to the ground state, by detecting the particles coming from the weak decay of the hyperon from the ground state and measuring their kinetic energies.

\subsection{Electromagnetic Form Factors of the Proton in the Timelike Region}

The electromagnetic form factors of the proton in the time-like region can be extracted from the cross section for the process $\bar{p} p \rightarrow e^{+} e^{-}$. First order QED predicts:

$$
\frac{d \sigma}{d \cos \theta^{*}}=\frac{\pi \alpha^{2} \hbar^{2} c^{2}}{2 x s}\left[\left|G_{M}\right|^{2}\left(1+\cos ^{2} \theta^{*}\right)+\frac{4 m_{p}^{2}}{s}\left|G_{E}\right|^{2}\left(1-\cos ^{2} \theta^{*}\right)\right]
$$

with $G_{E}$ and $G_{M}$ the electric and magnetic form factors, respectively. Data at high $Q^{2}$ are crucial 


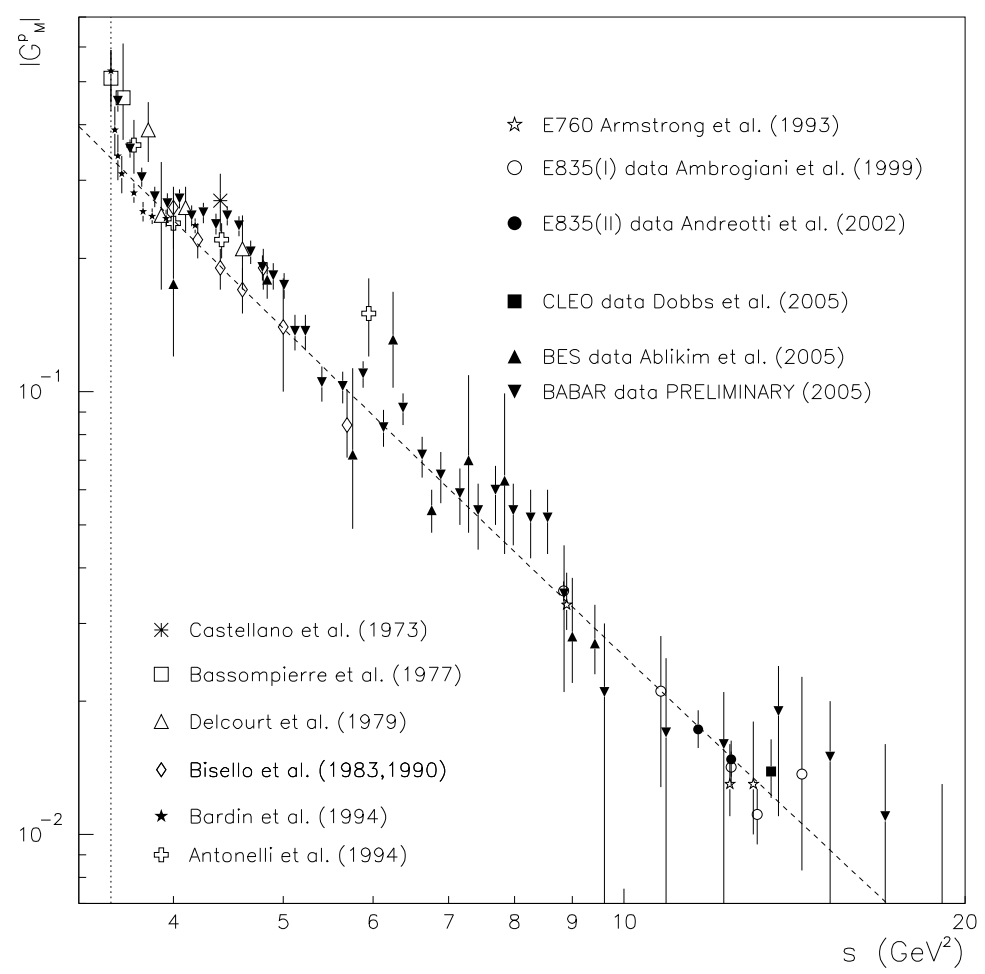

Figure 5: Measurements of the proton magnetic form factor $\left|G_{M}\right|$ in the time-like region.

to test their $Q^{2}$ behaviour and the space-like-time-like equality for corresponding $Q^{2}$.

The proton time-like form factors have been measured by several experiments in the low $Q^{2}$ region down to threshold. At high $Q^{2}$ the only measurements have been achieved by E760 and E835 at Fermilab [30] up to $Q^{2} \approx 15 \mathrm{GeV}^{2}$ (see also Fig. 5). However, due to limited statistics $\left|G_{M}\right|$ and $\left|G_{E}\right|$ have not been measured separately and could only be extracted using the assumption $\left|G_{E}\right|=\left|G_{M}\right|$. Recently new measurements of $\left|G_{M}\right|$ have been obtained by the BaBar collaboration [31] using Initial State Radiation.

In PANDA it will be possible to determine the form factors over the widest $Q^{2}$ range ever covered by a single experiment, from threshold to $20 \mathrm{GeV}^{2}$ or above, depending on beam time availability. Due to much higher statistics it will be possible to measure $\left|G_{M}\right|$ and $\left|G_{E}\right|$ separately. The real and imaginary parts of these time-like form factors could be determined with polarized beam and/or target. A modulation of the azimuthal distribution will certainly help disentangling the electric and the magnetic parts and give an information on the relative phase, even if this will be most likely limited by the luminosity to moderate $Q^{2}$ values. Most of the anticipated measurements could be run together with other programs. The proximity of resonance peaks should be avoided to reduce systematic uncertainties.

\section{The PANDA Detector}

In order to carry out the physics program discussed above the PANDA detector must fulfil a number of requirements: it must provide (nearly) full solid angle coverage, it must be able to handle 
high rates $\left(2 \times 10^{7}\right.$ annihilations/s) with good particle identification and momentum resolution for $\gamma, \mathrm{e}, \mu, \pi, \mathrm{K}$ and $\mathrm{p}$. Additional requirements include vertex reconstruction capability and, for charmonium, a pointlike interaction region, efficient lepton identification and excellent calorimetry (both in terms of resolution and of sensitivity to low-energy showers).

A schematic view of the PANDA detector is shown in Fig. 6. The antiprotons circulating in the HESR hit an internal hydrogen target (either pellet or cluster jet), while for the nuclear part of the experimental program wire or fiber targets will be used. The apparatus consists of a central detector, called Target Spectrometer (TS) and a Forward Spectrometer (FS).

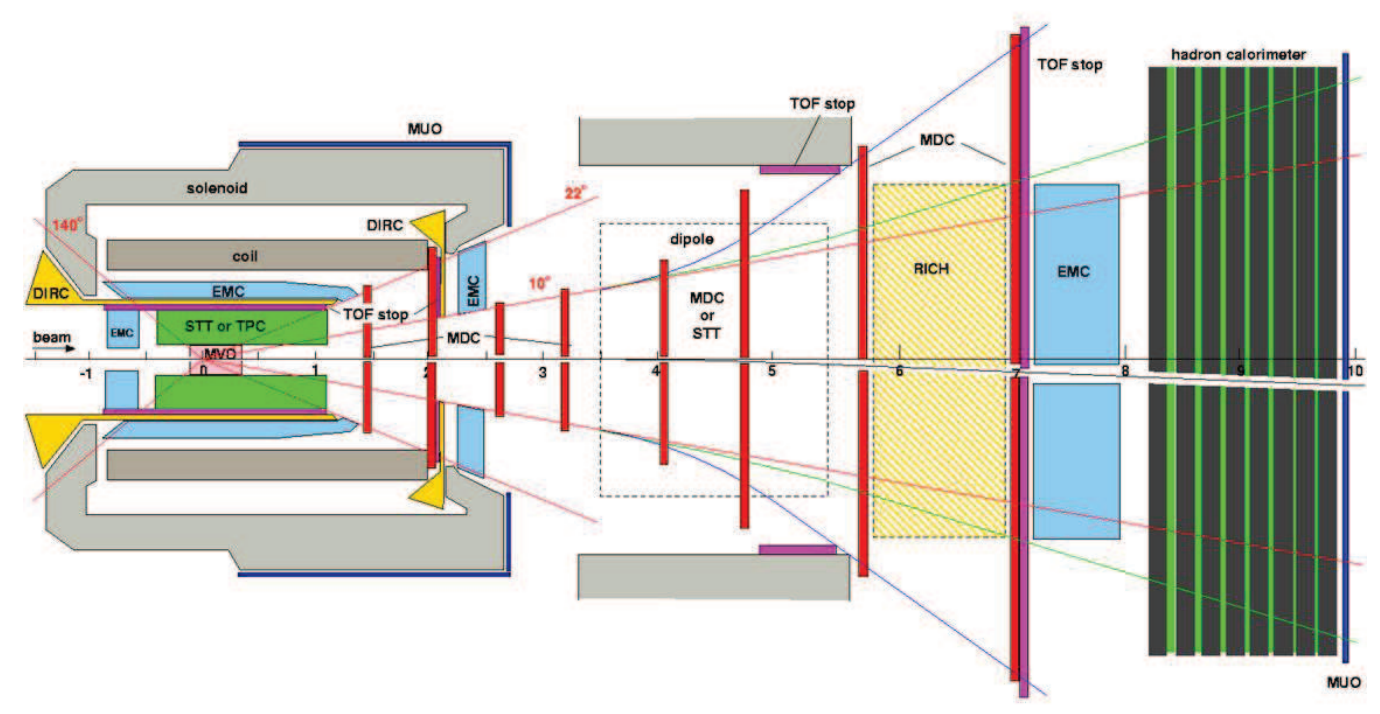

Figure 6: Schematic view of the PANDA detector.

The TS, for the measurement of particles emitted at laboratory angles larger than $5^{\circ}$, will be located inside a solenoidal magnet which provides a field of $2 \mathrm{~T}$. Its main components will be a microvertex silicon detector, a central tracker (either a straw tube detector or a time projection chamber), an inner time-of-flight telescope, a cylindrical DIRC (Detector of Internally Reflected Light) for particle identification, an electromagnetic calorimeter consisting of $\mathrm{PbWO}_{4}$ crystals, a set of muon counters and of multiwire drift chambers.

The FS will detect particles emitted at polar angles below $10^{\circ}$ in the horizontal and $5^{\circ}$ in the vertical direction. It will consist of a $2 \mathrm{~T} \cdot \mathrm{m}$ dipole magnet, with tracking detectors (straw tubes or multiwire chambers) before and after for charged particle tracking. Particle identification will be achieved by means of Čerenkov and time-of-flight detectors. Other components of the FS are and electromagnetic and a hadron calorimeter.

All detector components are currently being developed within a very active R\&D program. This continued development implies that the choice has not yet been finalized for all detector elements. 


\section{The PAX Physics Program}

The possibility to polarize antiprotons at FAIR will allow unique access to a number of new fundamental physics observables, which cannot be studied at other facilities or at the HESR without transverse polarization of protons and/or antiprotons.

The PAX experiment will be dedicated primarily to the study of nucleon structure using polarized antiproton-proton collisions, both in fixed target and in the collider mode.

The experimental program consists of a series of unique measurements; in this paper we will discuss the following:

- the first measurement of the transversity distribution, the last leading-twist missing piece of the QCD description of the partonic structure of the nucleon;

- the investigation of (transverse) single spin asymmetries;

- the measurement of the moduli and phases of the electromagnetic form factors of the proton in the time-like region.

\subsection{Transversity in Polarized Deep Inelastic Scattering}

In QCD the internal structure of hadrons is described by a hierarchy of parton correlation functions, the simplest of which are the parton distributions. Three functions are needed at leading twist for a full description of the parton structure of the nucleon:

- the unpolarized quark distribution $f_{1}(x)$;

- the helicity distribution $g_{1}(x)$, describing the quark longitudinal polarization inside a longitudinally polarized nucleon;

- the transversity distribution $h_{1}(x)$, describing the quark transverse polarization inside a transversely polarized nucleon (at infinite momentum).

Whereas the unpolarized distributions are well known, and more and more information is becoming available on $g_{1}$, nothing is known experimentally on the nucleon transversity distribution. The reason is that transversity is a chiral-odd function and, as a consequence, it cannot be measured in inclusive deep inelastic scattering: since strong and electroweak interactions conserve chirality, $h_{1}$ cannot appear alone, but it must be coupled to another chiral-odd quantity.

This is possible in two processes: semi-inclusive deep inelastic scattering (SIDIS), where, however, $h_{1}$ couples to a new, unknown fragmentation function, the Collins function [32], and in polarized Drell-Yan processes, which are sensitive to the product of two transversity distributions $h_{1}(x) \times h_{1}(x)$ and which therefore allow the direct measurement of $h_{1}$.

Experimentally the most direct way to measure transversity is the measurement of the double transverse spin asymmetry $A_{T T}$ in Drell-Yan processes with transversely polarized beam and target:

$$
A_{T T}=\frac{d \sigma^{\uparrow \uparrow}-d \sigma^{\uparrow \downarrow}}{d \sigma^{\uparrow \uparrow}+d \sigma^{\uparrow \downarrow}}=a_{T T} \frac{\sum_{q} e_{q}^{2} h_{1}^{q}\left(x_{1}, M^{2}\right) h_{1}^{\bar{q}}\left(x_{2}, M^{2}\right)}{\sum_{q} e_{q}^{2} f_{1}^{q}\left(x_{1}, M^{2}\right) f_{1}^{\bar{q}}\left(x_{2}, M^{2}\right)}
$$



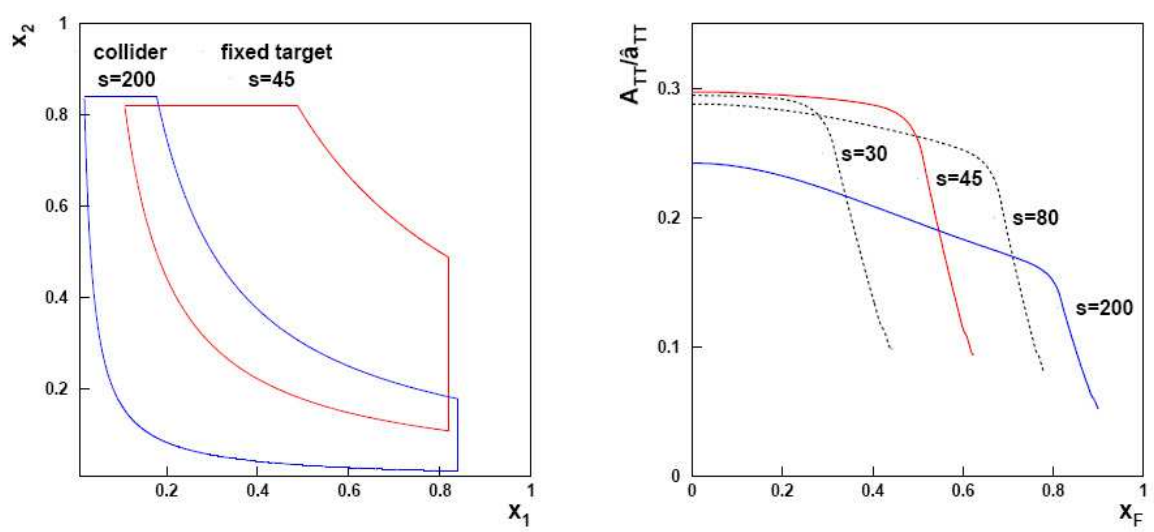

Figure 7: Left: the $\left(x_{1}, x_{2}\right)$ kinematical region covered by PAX. Right: The expected asymmetry as a function of $x_{F}$.

where $q=u, \bar{u}, d, \bar{d}, \ldots, \mathrm{M}$ is the invariant mass of the dilepton pair and $a_{T T}$ is the double spin asymmetry of the QED elementary process $q \bar{q} \rightarrow l^{+} l^{-}$.

The measurement of $A_{T T}$ can be carried out using polarized $p p$ interactions, as is being planned at RHIC [33]. In this case one measures the product of two transversity distributions, one for a quark and one for an antiquark (both from the proton). At RHIC energies one expects $x_{1} x_{2}=M^{2} / s \approx 10^{-3}$, which means that mainly the sea quark content of the proton will be explored, where polarization is likely to be small. In addition in the small $\mathrm{x}$ region explored by RHIC $h_{1}$ is much smaller than the corresponding values of $f_{1}$ and $g_{1}$. All this makes the double spin asymmetry expected at RHIC of the order of a few percent or less [34].

The PAX experiment, on the other hand, will measure the transverse double spin asymmetry using the polarized Drell-Yan process $\bar{p}^{\uparrow} p^{\uparrow} \rightarrow l^{+} l^{-} X$, in which a transversely polarized beam will hit a transversely polarized proton target (fixed target mode) or a transversely polarized proton beam (fixed target mode). In the hypothesis of u-quark dominance $\left|h_{1 u}\right|>>\left|h_{1 d}\right|$ and the expression for $A_{T T}$ for the $\bar{p} p$ process becomes:

$$
A_{T T} \approx a_{T T} \frac{h_{1 u}\left(x_{1}, M^{2}\right) h_{1 u}\left(x_{2}, M^{2}\right)}{u\left(x_{1}, M^{2}\right) u\left(x_{2}, M^{2}\right)}
$$

The combination of the fixed target and collider modes will allow PAX to explore ranges of $\mathrm{s}$ between $30 \mathrm{GeV}^{2}$ and $200 \mathrm{GeV}^{2}$ and $\mathrm{M}^{2}$ values between $4 \mathrm{GeV}^{2}$ and $100 \mathrm{GeV}^{2}$, where the values of $A_{T T}$ are expected to be large.

The $\left(x_{1}, x_{2}\right)$ kinematical regions covered by PAX in the two operational modes are illustrated in Fig. 7, left side. In fixed target mode one has $x_{1} x_{2}=M^{2} / s \approx 0.2-0.3$, which means that the dominant contribution will come from quarks and antiquarks with large $\mathrm{x}$, that means valence quarks and antiquarks for which $h_{1}$ is expected to be large. The plot on the right shows the expected values of $A_{T T}$ as a function of $x_{F}=x_{1}-x_{2}$ for different values of $s$ and $Q^{2}=16 \mathrm{GeV}^{2}$. The curves show that even at the highest $s$ values the double spin asymmetry remains above $20 \%$ for almost all the range of $x_{F}$. 
The measurement of the double spin asymmetry in the polarized $\bar{p} p$ Drell-Yan process will offer a unique opportunity to measure the transversity distributions of the proton for valence quarks.

\subsection{Single Spin Asymmetries}

In addition to transversity there are several other spin observables which can be measured by PAX, some of which might be within reach even before antiproton polarization is achieved.

An example of this kind of observables is given by single-spin asymmetries (SSA). Several mechanisms can generate SSA, both in the Parton Density Function (Sivers [35] and BoerMulders [36] effects) and in the fragmentation function (Collins effect [32]). Let us consider as an example the transverse SSA:

$$
A_{N}=\frac{d \sigma^{\uparrow}-d \sigma^{\downarrow}}{d \sigma^{\uparrow}+d \sigma^{\downarrow}}
$$

Large, unexpected transverse SSA have been observed by many experiments in $p^{\uparrow} p \rightarrow \pi X$ and $\bar{p}^{\uparrow} p \rightarrow \pi X$ : values as high as $40 \%$ have been measured in experiments with center of mass energies ranging from $6.6 \mathrm{GeV}$ to $200 \mathrm{GeV}$ [37].

The PAX experiment can provide completely new insight into the understanding of this phenomenon by studying SSA in D meson production $\left(\bar{p}^{\uparrow} p \rightarrow D X\right.$ or $\left.\bar{p} p^{\uparrow} \rightarrow D X\right)$, a process which allows to disentangle the Sivers from the Collins effect. At the PAX collider energy, for a final state $\mathrm{D}$ with a transverse momentum of about $2 \mathrm{GeV} / \mathrm{c}$ the dominant subprocess is $q \bar{q} \rightarrow c \bar{c}$ followed by the fragmentation of the $c$ quark into a charmed meson. In this elementary annihilation process there is no transverse spin transfer and the final $c$ and $\bar{c}$ are not polarized. Therefore there cannot be any contribution to the SSA from the Collins mechanism. Therefore a measurement of the SSA in $\bar{p}^{\uparrow} p \rightarrow D X$ or $\bar{p} p^{\uparrow} \rightarrow D X$ would allown a clean access to the Sivers function $f_{1 T}^{\perp}$.

In addition to that, and in conjunction with SSA data from the HERMES collaboration [38], the PAX measurement of SSA in Drell-Yan production on transversely polarized protons can for the first time provide a test of the theoretical prediction [39] of the sign reversal of the Sivers function from SIDIS to Drell-Yan processes:

$$
f_{1 T}^{\perp}(D Y)=-f_{1 T}^{\perp}(S I D I S)
$$

This measurement is of crucial importance for our current understanding of T-odd parton distributions and of SSA within QCD.

\subsection{Electromagnetic Form Factors of the Proton in the Timelike Region}

The measurement of the electromagnetic form factors of hadrons provides fundamental information on their structure and internal dynamics. For the proton the form factors have been measured both in the space-like and in the time-like regions over an extended range of $q^{2}$. There are, however, several outstanding issues. In the space-like regions recent measurents carried out at Jefferson Laboratory using the scattering process $\vec{e} p \rightarrow e \vec{p}$ with electron-to-proton polarization transfer have shown that the ratio of the Sachs form factors $G_{E}\left(q^{2}\right) / G_{M}\left(q^{2}\right)$ decreases monotonically with increasing $Q^{2}=-q^{2}$ [40], in strong contradiction with $G_{E} / G_{M}$ scaling observed in measurements carried out with the Rosenbluth separation method. In the time-like region, where 


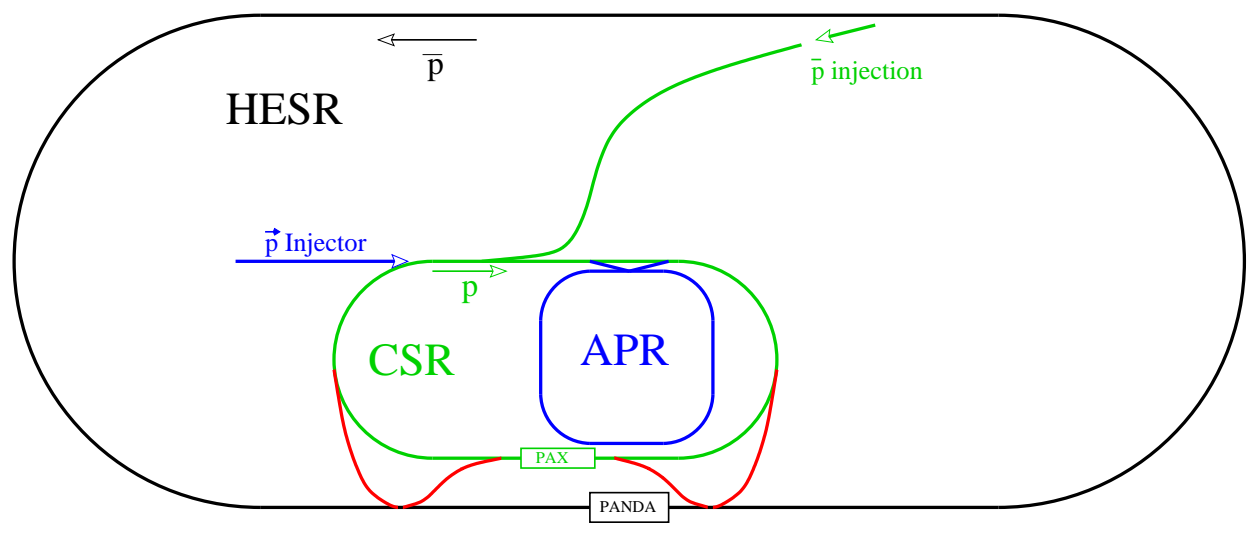

Figure 8: The HESR accelerator setup for PAX.

the form factors are complex, only the moduli have been measured. Furthermore, because of limited statistics, no experiment so far has been able to measure $\left|G_{E}\right|$ and $\left|G_{M}\right|$ separately and the magnetic form factor has been derived from the measured cross section assuming $\left|G_{E}\right|=\left|G_{M}\right|$ or $\left|G_{E}\right|=0$. The space-like and time-like form factors are connected by dispersion relations.

The relative phase of the proton form factors in the time-like region can be derived from the measurement of the transverse single spin asymmetry in antiproton annihilation on transversely polarized protons $\bar{p} p^{\uparrow} \rightarrow e^{+} e^{-}$. The asymmetry $\mathscr{A}_{y}$ is given by:

$$
\mathscr{A}_{y}=\frac{\sin 2 \theta \operatorname{Im} G_{E}^{*} G_{M}}{\left[\left(1+\cos ^{2} \theta\right)\left|G_{M}\right|^{2}+\sin ^{2} \theta\left|G_{E}\right|^{2} / \tau\right] \sqrt{\tau}}
$$

where $\tau=q^{2} / 4 m_{p}^{2}$ and $\theta$ is the scattering angle. The PAX experiment plans to measure the transverse single spin asymmetry in the collisions of an unpolarized antiproton beam with a transversely polarized, internal proton target. The maximum beam momentum of $3.5 \mathrm{GeV} / \mathrm{c}$ will allow the measurement over a wide kinematical range, from close to threshold up to $\mathrm{Q}^{2}=8.5 \mathrm{GeV}^{2}$.

In addition to that, $G_{E}-G_{M}$ separation can be achieved with the measurement of the transverse double spin asymmetry in $\bar{p}^{\uparrow} p^{\uparrow} \rightarrow l^{+} l^{-}$, using a method which is complementary to the one used by PANDA and discussed in section 3.5, thus providing a test of Rosenbluth separation in the time-like region.

\section{The PAX experimental setup}

The overall machine setup of the HESR complex needed for the PAX experiment is shown schematically in Fig. 8. The Antiproton Polarizer (APR) will polarize antiprotons at kinetic energies around $50-250 \mathrm{MeV}$ to be injected into the other rings. The Cooler Synchrotron Ring (CSR) will store protons or antiprotons with momenta up to $3.5 \mathrm{GeV} / \mathrm{c}$. The small straight section of this ring will effectively provide a second interaction point where the PAX detector could be installed, thus allowing PANDA and PAX to be run in parallel.

The PAX scientific program discussed in the previous sections will be carried out in two phases: 
- in phase I a beam of unpolarized antiprotons with momentum up to $3.5 \mathrm{GeV} / \mathrm{c}$ in the CSR will collide with an internal hydrogen target. This phase will be mainly dedicated to the measurement of the time-like proton form factors discussed in the previous section. Furthermore, by detecting antiprotons scattered in the backward direction, it will be possible to study hard scattering at large $t$.

- in phase II a beam of polarized antiprotons from $1.5 \mathrm{GeV} / \mathrm{c}$ to $15 \mathrm{GeV} / \mathrm{c}$ circulating in the HESR will collide with a beam of polarized protons with momentum up to $3.5 \mathrm{GeV} / \mathrm{c}$ circulating in the CSR. This phase will be dedicated to the first direct measurement of the transversity distribution $h_{1}$. With suitable combinations of the energies of the two colliding beams this setup will allow a measurement of $h_{1}$ in the valence region of $x>0.05$ with corresponding $\mathrm{Q}^{2}=4 \ldots 100 \mathrm{GeV}^{2}$.

The PAX detector [41] is being developed by means of Monte Carlo studies aiming at an optimization of the achievable performance. The detector will be designed for a collider, but it will be compatible with the fixed target running mode. In the present concept it will employ a toroidal magnet and it will be optimized for the detection of $e^{+} e^{-}$pairs from the Drell-Yan process.

\section{Antiproton polarization}

The feasibility and success of the PAX experimental program will depend crucially on the capability to produce a transversely polarized antiproton beam, with polarizations in the range 0.2 0.3. The most promising approach towards this goal is the use of the spin filtering technique, based on the interaction of unpolarized antiprotons with a polarized hydrogen target. This technique has been successfully tested and confirmed experimentally with protons by the FILTEX experiment [42]. However the interpretation of the FILTEX data is still somewhat controversial, and there is no universally accepted explanation as to why spin filtering works. This highlights the need for further experimental tests. In addition to that, there is no data to predict what level of polarization could be achieved with antiprotons. For these reasons the PAX collaboration is planning to conduct a series of test experiments, first with protons at the COSY ring in Jülich, then with antiprotons at the Antiproton Decelerator at CERN. The aim of these tests is to fully understand the mechanism of proton and antiproton polarization and to measure the level of polarization which could be achieved for an antiproton beam.

\section{Conclusions}

The availability of high-intensity, cooled antiproton beams at FAIR will make it possible to perform a very rich experimental program.

The PANDA experiment will perform high-precision hadron spectroscopy from $\sqrt{s}=2.25 \mathrm{GeV}$ to $\sqrt{s}=5.5 \mathrm{GeV}$ and produce a wealth of new results:

- precision measurement of the parameters of all charmonium states, both below and above open charm threshold, with the possible discovery of the missing states (e.g. the D-wave states), which will lead to a full understanding of the charmonium spectrum; 
- the observation/discovery of glueballs and hybrids, particularly in the mass range between 3 and $5 \mathrm{GeV} / \mathrm{c}^{2}$, yielding new insights into the structure of the QCD vacuum;

- the measurement of mass shifts of charmonium and open charm mesons in nuclear matter, related to the partial restoration of QCD chiral symmetry in a dense nuclear medium;

- the discovery and spectroscopy of new double-strange hypernuclei, providing new insights in the hyperon-hyperon and hyperon-nucleon interaction;

- high-statistics measurement of the electromagnetic form factor of the proton in the timelike region over a wide $\mathrm{Q}^{2}$ range from near threshold to about $20 \mathrm{GeV}^{2}$, with the separate determination of the electric and magnetic form factors (for the low $\mathrm{Q}^{2}$ region) by measuring the angular distribution.

The possibility to polarize antiprotons will allow the PAX experiment to make new, unique measurements of single- and double-spin observables:

- the first measurement of the transversity distribution of the valence quarks in the proton;

- new measurements of single-spin asymmetries, leading to a better understanding of the Sivers and Collins mechanisms and of the origin of T-odd parton distributions;

- the first determination of the relative phase of the electric and magnetic form factors of the proton in the time-like region.

All these new measurements will make it possible to achieve a very significant progress in our understanding of QCD and the strong interaction. We are looking forward to many years of exciting hadron physics at FAIR.

\section{References}

[1] J. J. Aubert et al., Phys. Rev. Lett. 33(1974)1404;

J. E. Augustin et al., Phys. Rev. Lett. 33(1974)1406.

[2] P.Dalpiaz, Proceedings of the $f$ irst LEAR workshop, Karlsruhe, Germany, edited by H. Poth (1979).

[3] M. Andreotti et al., Nucl. Phys.B717(2005)34.

[4] W.-M. Yao et al., J. of Phys.G33(2006)1.

[5] C. Edwards et al., Phys. Rev. Lett. 48(1982)70.

[6] S. K. Choi et al., Phys. Rev. Lett. 89(2002)102001.

[7] D. M. Asner et al., Phys. Rev. Lett. $92(2004) 142001$.

[8] B. Aubert et al., Phys. Rev. Lett. 92(2004)142002.

[9] T. A. Armstrong et al., Phys. Rev. Lett. 69(1992)2337.

[10] M. Andreotti et al., Phys. Rev. D 72(2005)032001.

[11] J. L. Rosner et al.,Phys. Rev. Lett. 95(2005)102003. 
[12] J. Z. Bai et al., Phys. Rev. Lett. 84(2000)594200;

J. Z. Bai et al., Phys. Rev. Lett. 88(2002)101802.

[13] S. Uehara et al., Phys. Rev. Lett.96(2006)082003.

[14] K. Abe et al., Phys. Rev. Lett.94(2005)182002.

[15] D. Thompson et al., Phys. Rev. Lett.79(1997)1630;

G. Adams et al., Phys. Rev. Lett.81(1998)5760.

[16] A. Abele et al., Phys. Lett.B 423(1998)175.

[17] J. Reinnarth et al., Nucl. Phys.A 692(2001)268c.

[18] C. Amsler et al., Phys. Lett. B 355(1995)425.

[19] C. Morningstar and M. Peardon, Phys. Rev. D 60(1999)34509.

[20] M. Bargiotti et al., Phys. Lett.B 561(2003)233.

[21] F. E. Close and A. Kirk, Phys. Lett. B483(2000)345.

[22] H. Geissel et al., Phys. Rev. Lett.88(2002)122301;

H. Geissel et al., Phys. Lett.B549(2002)64;

H. Geissel et al., Phys. Rev. Lett.92(2002)072302.

[23] M. Nekipelov et al., Phys. Lett.B540(2002)207;

Z. Rudy et al., Eur. Phys. J.A15(2002)303;

Y. Shin et al., Phys. Rev. Lett.81(1998)1576;

R. Barth et al., Phys. Rev. Lett.78(1997)4007;

F. Laue et al., Phys. Rev. Lett.82(1999)1640;

P. Crochet et al., Phys. Lett.B486(2000)6;

K. Wisniewski et al., Eur. Phys. J.A9(2000)515.

[24] W. Klingl et al., Nucl. Phys.A624(1997)527.

[25] S. Lee and C. Ko, Phys. Rev.C67(2003)038202;

S. Lee, Proceedings of Int. Workshop "Hadron 2003", edited by H. K. E. Klempt and H. Orth., Aschaffenburg, 2004.

[26] A. Sibirtsev, K. Tsushima and A. Thomas, Eur. Phys. J.A6(1999)351.

[27] A. Hayashigaki, Phys. Lett.B487(2000)96;

B. Friman, S. Lee and T. Song, Phys. Lett.B548(2002)153.

[28] M. Danysz and J. Pniewski, Phil. Mag.44(1953)348).

[29] M. Danysz,Nucl. Phys.49(1963)121.

[30] T. A. Armstrong et al., Phys. Rev. Lett.70(1993)1212;

M. Ambrogiani et al., Phys. Rev.D 60(1999)032002;

M. Andreotti et al.,Phys. Lett.B559(2003)20.

[31] B. Aubert et al., Phys. Rev.D73(2006)051105.

[32] J. C. Collins, Nucl. Phys.B396(1993)161.

[33] W. Vogelsang, QCD spin physics: status and prospects for RHIC, Pramana 63(2004)1251. 
[34] V Barone, T. Calarco and A. Drago, Phys. Rev.D56(1997)527;

O. Martin et al., Phys. Rev.D57(1998)3084;

O. Martin et al., Phys. Rev.D60(1999)117502.

[35] D. Sivers, Phys. Rev. Lett.D41(1990)83;

D. Sivers, Phys. Rev. Lett.D43(1991)261.

[36] D. Boer and P.J. Mulders, Nucl. Phys.B569(2000)505.

[37] K. Krueger et al., Phys. Lett.B459(1999)412;

D. L. Adams et al., Phys. Lett.B264(1991)462);

A. Bravar et al., Phys. Rev. Lett.77(1996)2626;

J. Adams et al., Phys. Rev. Lett.92(2004)171801.

[38] A. Airapetian et al., Phys. Rev. Lett.84(2000)4047;

A. Airapetian et al., Phys. Rev. Lett.90(2003)092002;

A. Airapetian et al., Phys. Rev.D 64(2001)097101;

K. Rith, Progr. in Part. and Nucl. Phys.49(2002) 245.

[39] A. Airapetian et al., Phys. Rev. Lett.94(2005)012002.

[40] M. K. Jones et al., Phys. Rev. Lett.84(2000)1398;

O. Gayou et al., Phys. Rev. Lett.88(2002)092301;

O. Gayou et al., Phys. Rev.C64(2001)038202.

[41] PAX technical proposal, http://www.fz-juelich.de/ikp/pax.

[42] F. Rathmann et al., Phys. Rev. Lett.71(1993)1379. 